\title{
A NOTE ON THE BOREL STRUCTURE OF A METRIZABLE CHOQUET SIMPLEX AND OF ITS EXTREME BOUNDARY
}

\author{
ERIK M. ALFSEN
}

V. Klee was the first to study the monotone class generated by the convex closed sets [12]. This type of construction was transferred from convex sets to affine functions in [2]. Here it was shown that the simplexes are exactly those convex compact sets for which any two mutually singular boundary measures admit a separator from the monotone class $\mathscr{A}$ generated by semi-continuous affine functions.

Generally $\mathscr{A}$ is contained in the class of all bounded affine Borel functions, and the two classes need not be identical. (Cf. [2] and also Proposition 1 of the present paper. The appropriate counter-example was given by G. Choquet for a somewhat different purpose [8].)

In the present paper it is shown that the class $\mathscr{A}$ over a metrizable simplex $K$ corresponds biuniquely to the class of all bounded Borel functions on the extreme boundary $\partial_{e} K$ of $K$. Specifically, every bounded Borel function on $\partial_{e} K$ can be extended uniquely to a function of class $\mathscr{A}$ on $K$ (Theorem 2). Moreover, the class $\mathscr{A}$ consists of exactly those bounded Borel functions on $K$ for which the "barycenter formula" is valid (Theorem 2, Corollary 1).

Sets of the form $f^{-1}(0)$ with $f \in \mathscr{A}^{+}$, are said to be faces of class $\mathscr{A}$. Generally, every closed face is of class $\mathscr{A}$, and every face of class $\mathscr{A}$ is a Borel face. In a metrizable simplex $K$, the faces of class $\mathscr{A}$ form a $\sigma$-complete Boolean algebra under convex combination, intersection, and passage to the complementary face (Theorem 3). In particular, $K$ is direct convex sum of $F$ and $F^{\prime \prime}$ for every face $F$ of class $\mathscr{A}$. (Cf. [3] for the definition of the "complementary face" $F^{\prime}$.)

The concept of an $\mathscr{A}$-face provides a geometric criterion for absolute continuity of representing boundary measures $\mu_{x}$ of points $x$ of a metrizable simplex with respect to a given boundary measure $\mu$. Specifically, $\mu_{x} \ll \mu$ if and only if $x$ is contained in every $\mathscr{A}$-face containing the barycenter of $\mu$ (Theorem 4).

Received June 1, 1966.

Math. Scand. $19-11$ 
The above results lean heavily on the fact that the class $\mathscr{A}$ over a metrizable simplex is a (conditionally) $\sigma$-complete vector lattice (Theorem 1). This result is not entirely obvious, as the (generating) class of continuous affine functions need not be a vector lattice. In this connection we recall that the linear space of continuous affine functions on a convex set $K$ is lattice-ordered if and only if $K$ is a simplex with closed extreme boundary, and that it enjoys the F. Riesz decomposition property if and only if $K$ is a general simplex. The first result is due to $H$. Bauer [5] and the second to J. Lindenstrauss [13] and Z. Semadeni [15]. An elegant proof of the latter result was given by D. A. Edwards in $[10]$.

\section{Definitions and basic properties.}

In the sequel $K$ shall be a convex compact subset of a locally convex (Hausdorff) space $E$ over the reals. The extreme boundary of $K$ (i.e. the set of extreme points) is denoted by $\partial_{e} K$, and for every $x \in K$ the set of positive normalized boundary measures with barycenter $x$ is denoted by $\mathfrak{M}_{x}^{+}$. (For the definition of a "boundary measure" ef. e.g. $[1$, p. 98].) Recall that $K$ is a simplex if and only if $\mathfrak{M}_{x}^{+}$has a unique member $\mu_{x}$ for every $x$ in $K$.

The linear space of all $K$-restrictions of (real valued) continuous affine functions on $E$ is denoted by $\mathscr{H}$ and the linear spaces of u.s.c. and l.s.c. affine functions on $K$ are denoted by $\mathscr{F}$ and $\mathscr{G}$, respectively. The smallest class of real valued functions on $K$ which contains $\mathscr{F}$ and $\mathscr{G}$ and is closed under pointwise limits of (pointwise bounded) monotone sequences, is denoted by $\mathscr{A}$. The following property of $\mathscr{A}$ is essentially a restatement of Proposition 2 of [2].

Proposition 1. Every member $f$ of $\mathscr{A}$ is a bounded affine Borel function for which the barycenter formula is valid, i.e. for every positive normalized measure $\mu$ on $K$ with barycenter $x$

$$
f(x)=\int f d \mu .
$$

Proof. Clearly $f$ is an affine Borel function. By Proposition 2 of [2], $f$ is $\mu$-integrable for every positive normalized measure $\mu$, and the formula (1.1) is valid. Assume $f$ unbounded. Then there exists a sequence $\left\{x_{n}\right\}$ of points in $K$ such that $f\left(x_{n}\right)>n^{-1}$ for $n=1,2, \ldots$. Let $\mu$ be the measure with point-masses $n^{-2}$ at $x_{n}$ for $n=1,2, \ldots$. Now $\mu$ is a positive measure on $K$ with respect to which $f$ is non-integrable, contrary to the above statement. 
For later references we note the following "metrizable" version of a well-known general property (cf. e.g. [9, p. 140] and [2, Prop. 1]).

Proposition 2. If $K$ is a metrizable convex compact set and if $f$ is an u.s.c. concave function on $K$, then there is a sequence $\left\{h_{n}\right\}$ from $\mathscr{H}$ such that $f=\inf _{n} h_{n}$. If $f$ is affine, i.e. if $f \in \mathscr{F}$, then the sequence $\left\{h_{n}\right\}$ may be chosen to be descending. Clearly, the dual statements are valid under the dual hypotheses.

Proof. By a standard argument (based on Hahn-Banach separation in $E \times \mathrm{R}$ ), one may construct a family $\left\{h_{\alpha}\right\}_{\alpha \in A}$ from $\mathscr{H}$ such that $\inf _{\alpha} h_{\alpha}=f$ (cf. e.g. [13, ch. 3]). Making use of a countable base of open sets for $K$, one may render the index set $A$ countable. Note that the functions $h_{\alpha}$ constructed above can be chosen as strict majorants of $f$, in the sense that $h_{\alpha}(x)>f(x)$ for all $x$ in $K$. Now it is known that the family of strict majorants of class $\mathscr{H}$ of some u.s.c. affine function is directed downward (cf. e.g. [2, Prop. 1]). This yields the last part of the proposition.

Corollary. If $K$ is a metrizable convex compact set, then $\mathscr{F}$ is equal to the class $\mathscr{H}_{\delta}$ of all pointwise limits of descending sequences from $\mathscr{H}$, and $\mathscr{G}$ is equal to the corresponding dual class $\mathscr{H}_{\sigma}$. Moreover, $\mathscr{A}$ is the smallest class of real valued functions which contains $\mathscr{H}$ and is closed under pointwise limits of monotone sequences.

It should be noted that the barycenter formula imposes certain regularity properties on the functions of class $\mathscr{A}$ at points off the extreme boundary. In this connection we recall that the face generated by a point $x$ of $K$ is the union of all sets

$$
D(x, \alpha)=(\alpha x-(\alpha-1) K) \cap K, \quad \alpha \geqq 1 .
$$

In particular, $x \in \partial_{e} K$ if and only if $D(x, \alpha)=\{x\}$ for all $\alpha \geqq 1$.

Proposition 3. A pointwise bounded, monotone sequence $\left\{f_{n}\right\}$ of functions of class $\mathscr{A}$ on a simplex $K$ converges uniformly on every set $D(x, \alpha), x \in K$, $\alpha \geqq 1$. Consequently, every function of class $\mathscr{A}$ on $K$ has a continuous restriction to every set $D(x, \alpha)$.

Proof. If $y \in D(x, \alpha)$, then there is a point $z \in K$ such that

and hence

$$
y=\alpha x-(\alpha-1) z,
$$

$$
\mu_{y}=\alpha \mu_{x}-(\alpha-1) \mu_{z} \leqq \alpha \mu_{x} .
$$

It follows that $\mu_{y} \ll \mu_{x}$, and that the Radon-Nikodym derivative $k_{y}$ of $\mu_{y}$ with respect to $\mu_{x}$ satisfies $\left\|k_{y}\right\|_{\infty} \leqq \alpha$. 
Let $f$ be the pointwise limit of $\left\{f_{n}\right\}$. By definition $f \in \mathscr{A}$, and by the barycenter formula

$$
\left|f(y)-f_{n}(y)\right|=\left|\int\left(f-f_{n}\right) k_{y} d \mu_{x}\right| \leqq \alpha \int\left|f-f_{n}\right| d \mu_{x} .
$$

The right hand term of (1.3) is independent of the point $y$ of $D(x, \alpha)$, and it tends to zero as $n$ tends to infinity, by virtue of the Monotone Convergence Theorem. This proves the uniform convergence of $\left\{f_{n}\right\}$ over $D(x, \alpha)$.

Remark. In Proposition 3 it would be sufficient to assume convergence on some boundary set $B_{f}$ where $f \in \mathscr{C}(K)$, and in the metrizable case one might choose $B_{f}=\partial_{e} K$ (cf. e.g. $[1$, p. 98]).

\section{Lattice properties of $\mathscr{A}$.}

We shall prove that the class $\mathscr{A}$ over a metrizable simplex is lattice ordered in the natural ordering of functions. The non-metrizable case remains open.

ThEOREM 1. If $f$ and $g$ are functions of class $\mathscr{A}$ over a simplex $K$, then for every $x \in K$ :

$$
\begin{aligned}
& \int f \vee g d \mu_{x}=\sup \{\lambda f(y)+(1-\lambda) g(z) \mid x=\lambda y+(1-\lambda) z, 0 \leqq \lambda \leqq 1\}, \\
& \int f \wedge g d \mu_{x}=\inf \{\lambda f(y)+(1-\lambda) g(z) \mid x=\lambda y+(1-\lambda) z, 0 \leqq \lambda \leqq 1\},
\end{aligned}
$$

and the "sup" and "inf" values are effectively attained by appropriate convex combinations on $K$. If $K$ is metrizable, then $\mathscr{A}$ is a (conditionally) $\sigma$-complete vector lattice in the natural ordering of functions, and the lattice operations are given by the formulas

$$
\begin{aligned}
& (f \vee g)(x)=\int f \vee g d \mu_{x}, \\
& (f \wedge g)(x)=\int f \wedge g d \mu_{x} .
\end{aligned}
$$

Proof. 1) Consider first an arbitrary convex combination on $K$ :

Now

$$
x=\lambda y+(1-\lambda) z, \quad 0 \leqq \lambda \leqq 1 .
$$

$$
\mu_{x}=\lambda \mu_{y}+(1-\lambda) \mu_{z},
$$

and so by Proposition 1 


$$
\begin{aligned}
\int(f \vee g) d \mu_{x} & =\lambda \int(f \vee g) d \mu_{y}+(1-\lambda) \int(f \vee g) d \mu_{z} \\
& \geqq \lambda \int f d \mu_{y}+(1-\lambda) \int g d \mu_{z} \\
& =\lambda f(y)+(1-\lambda) g(z) .
\end{aligned}
$$

To prove that the equality sign of (2.5) subsists for some convex combination on $K$, we define

$$
A=\{y \mid f(y) \geqq g(y)\}, \quad B=\{y \mid f(y)<g(y)\} .
$$

If $\mu_{x}(A)=0$ or $\mu_{x}(B)=0$, then we may use the trivial convex combination $x=y=z$ to yield equality with the right hand term of (2.5) equal to $g(x)$ and $f(x)$, respectively. If $\mu_{x}(A) \neq 0$ and $\mu_{x}(B) \neq 0$, then we write $\mu_{x}(A)=\lambda$ and define

$$
\pi=\lambda^{-1}\left(\mu_{x}\right)_{A}, \quad \varrho=(1-\lambda)^{-1}\left(\mu_{x}\right)_{B} .
$$

Denoting the barycenters of $\pi$ and $\varrho$ by $y$ and $z$, respectively, we may write

$$
\begin{aligned}
\int f \vee g d \mu_{x} & =\int_{A} f d \mu_{x}+\int_{B} g d \mu_{x} \\
& =\lambda \int f d \pi+(1-\lambda) \int g d \varrho \\
& =\lambda f(y)+(1-\lambda) g(z) .
\end{aligned}
$$

The dual verification is similar.

2) Next assume $K$ metrizable and define the functions $f \vee g$ and $f \wedge g$ by the formulas (2.3), (2.4) for any two members $f, g$ of $\mathscr{A}$. For every $f \in \mathscr{A}$ define

$$
\mathscr{N}(f)=\{g \mid g \in \mathscr{A}, f \vee g \in \mathscr{A}, f \wedge g \in \mathscr{A}\} .
$$

It follows by application of the Monotone Convergence Theorem that $\mathscr{N}(f)$ is closed under pointwise limits of (pointwise bounded) monotone sequences. By the symmetry of the definition (2.6)

$$
f \in \mathscr{N}(g) \Leftrightarrow g \in \mathscr{N}(f) .
$$

Assume for a moment that $f, g \in \mathscr{H}$. By a known theorem (cf. e.g. $[9$, p. 141]), the envelopes of $f \vee g$ and $f \wedge g$ are given by

$$
\overline{(f \vee g)}(x)=\int f \vee g d \mu_{x}, \quad \overline{(f \wedge g)}(x)=\int f \wedge g d \mu_{x} .
$$

The functions $\overline{f \vee g}, f \wedge g$ are affine since $K$ is a simplex (cf: e.g. [9, p: 145]). Hence $f \vee g \in \overline{\mathscr{F}}$ and $f \wedge g \in \mathscr{G}$. It follows that 


$$
\mathscr{H} \subset \mathscr{N}(f) \text { for all } f \in \mathscr{H} \text {. }
$$

At this point we invoke the metrizability of $K$, by which we may apply the Corollary of Proposition 2 together with the closure properties of $\mathscr{N}(f)$ to yield the relation

$$
\mathscr{A} \subset \mathscr{N}(f) \text { for all } f \in \mathscr{H} \text {. }
$$

By (2.7), this may be transformed to

$$
\mathscr{H} \subset \mathscr{N}(g) \text { for all } g \in \mathscr{A},
$$

and by the same argument as above,

$$
\mathscr{A} \subset \mathscr{N}(g) \text { for all } g \in \mathscr{A} \text {. }
$$

Thus we have proved that $\mathscr{A}$ is closed under operations $f, g \frown f \vee g$ and $f, g \frown f \wedge g$.

Clearly $f \vee g$ is the least upper bound of $f$ and $g$ within $\mathscr{A}$, for if $k \in \mathscr{A}$ and $k \geqq f, k \geqq g$, then by Proposition 1

$$
k(x)=\int k d \mu_{x} \geqq \int f \vee g d \mu_{x}=(f \vee g)(x)
$$

for all $x \in K$. Similarly it can be seen that $f \wedge g$ is the greatest lower bound of $f$ and $g$ within $\mathscr{A}$. Hence $\mathscr{A}$ is a lattice in the natural ordering, with the prescribed lattice operations. Clearly $\mathscr{A}$ is a linear space; hence it is a vector lattice, and $\mathscr{A}$ is (conditionally) $\sigma$-complete by definition.

Remark. Assume for a moment that $K$ is located on a hyperplane not passing through the origin of $E$, and that $E=C-C$ where $C$ is the convex cone generated by $K$. By a well-known theorem of Choquet ([7], cf. also [9, p. 145]), $K$ is a simplex if and only if $C$ defines a lattice ordering in $E$. It is seen from the formulas (2.1), (2.2) that the lattice operations on $\mathscr{A}$ are those inherited from the vector lattice of order bounded (or "relatively bounded") linear functionals on $E$ (cf. e.g. [6, ch. II]).

\section{Boundary values for functions of class $\mathscr{A}$.}

Every bounded Borel function $f$ on the extreme boundary $\partial_{e} K$ of a metrizable simplex $K$ can be extended to an affine function $\tilde{f}$ on $K$ by the formula

$$
\tilde{f}(x)=\int f d \mu_{x}
$$

Proposition 4. If $f$ is a bounded u.s.c. function on the extreme boundary $\partial_{\sigma} K$ of a metrizable simplex $K$, then the extended function $\tilde{f}$ is of class $\mathscr{G}_{\delta}$. Dually if $f$ is bounded and 1.s.c., then $\tilde{f}$ is of class $\mathscr{F}_{\sigma}$. 
Proof. By a simple generalization of a theorem of M. Hervé, the upper envelope $\bar{f}$ of a bounded u.s.c, function $f$ satisfies the requirement $\bar{f}(x)=f(x)$ for all $x \in \partial_{e} K$. (Hervé's Theorem concerns continuous functions on $K$. The transition to semi-continuous functions on $\partial_{e} K$ can be found e.g. in the Lemma of [4].)

By Proposition 2, there is a sequence $\left\{h_{n}\right\}$ from $\mathscr{H}$ such that $\bar{f}=\inf _{n} h_{n}$. Define $g_{n}=h_{1} \wedge \ldots \wedge h_{n}$. Then $\left\{g_{n}\right\}$ is a descending sequence of continuous concave functions such that $g_{n} \searrow \bar{f}$. In particular

$$
\lim _{n \rightarrow \infty} g_{n}(x)=f(x), \quad x \in \partial_{e} K .
$$

By a known characterization of envelopes of continuous concave functions (cf. e.g. [9, p. 146]), and by the Monotone Convergence Theorem

$$
\lim _{n \rightarrow \infty} \underline{g_{n}}(x)=\lim _{n \rightarrow \infty} \int g_{n} d \mu_{x}=\int f d \mu_{x} .
$$

Hence we have proved $g_{n} \searrow f$. By a known property of simplexes (cf. e.g. $\left[9\right.$, p. 145]), $g_{n}$ is l.s.c. and affine. In other words $g_{n} \in \mathscr{G}$, and so $\tilde{f} \in \mathscr{G}_{\sigma}$. The dual verification is similar.

By definition, the class of Borel sets on the topological space $\partial_{e} K$ (induced topology) is the Boolean $\sigma$-algebra generated by closed (or open) sets, and it is equal to $\partial_{e} K \cap \mathscr{B}$ where $\mathscr{B}$ is the class of Borel sets on $K$. The bounded Borel (-measurable) functions form the smallest vector lattice of real valued functions which contains the characteristic functions of closed (or open) sets and is closed under pointwise limits of bounded monotone sequences.

Theorem 2. If $K$ is a metrizable simplex, then the restriction mapping $f \frown f / \partial_{e} K$ is a bijection of the class $\mathscr{A}$ onto the class of bounded Borel functions on $\partial_{e} K$, and its inverse is the mapping $f \frown \tilde{f}$ defined by (3.1).

Proof. By Proposition $1, f / \partial_{e} K$ is a bounded Borel function for every $f \in \mathscr{A}$, and every function of class $\mathscr{A}$ can be recovered from its boundary values by the integral formula (3.1). Hence it only remains to be proved that $\tilde{f} \in \mathscr{A}$ for every bounded Borel function $f$ on $\partial_{e} K$.

Let $\mathscr{B}_{0}$ be the class of all bounded Borel functions $f$ on $\partial_{e} K$ for which $\tilde{f} \in \mathscr{A}$. Clearly $\mathscr{B}_{0}$ is closed under linear operations, and by (2.3), (2.4), it is closed under pointwise lattice operations. By the Monotone Convergence Theorem $\mathscr{B}_{0}$ is closed under monotone limits of bounded sequences. By Proposition 4, $\mathscr{B}_{0}$ contains all characteristic functions of closed subsets of $\partial_{e} K$. Hence $\mathscr{B}_{0}$ comprizes all bounded Borel functions on $\partial_{e} K$, and the theorem is proved. 
Corolnary 1. A function $f$ on a metrizable simplex $K$ is of class $\mathscr{A}$ if and only if it is a bounded Borel function for which the barycenter formula is valid; i.e. if

$$
f(x)=\int f d \mu,
$$

whenever $\mu$ is a positive normalized measure with barycenter $x$.

Proop. The necessity follows from Proposition 1; and the sufficiency follows from Theorem 4, for if $f$ is a bounded Borel function on $K$ for which the barycenter formula is valid, then

$$
f=\overparen{f / \partial_{e} K} \in \mathscr{A} \text {. }
$$

Corollary 2. Every affine function of the first Baire class on a metrizable simplex is of class $\mathscr{A}$.

Proor. By a theorem of G. Choquet [8] (cf. [14, ch. 12] for a detailed proof), the barycenter formula is valid for every affine function of the first Baire class. Hence the conclusion follows from Corollary 1.

Corolmary 3. Let $K$ be a metrizable simplex. If $\mu$ and $v$ are mutually singular positive boundary measures on $K$, then there exists a function $f$ of class $\mathscr{A}$ such that $|f| \leqq 1$ and

$$
\mu(\{x \mid f(x) \leqq 0\})=\nu(\{x \mid f(x) \geqq 0\})=0 .
$$

If $\mu$ and $\nu$ are positive boundary measures such that $\nu \leqq \alpha \mu$ for some positive number $\alpha$, then the Radon-Nikodym derivative of $v$ with respect to $\mu$ admits a representative of class $\mathscr{A}$.

Proof. Clearly there exist bounded Borel functions on $\partial_{e} K$ with the required properties, which will prevail after extension of these functions to functions of class $\mathscr{A}$ on $K$.

Remark. The statement of the first part of Corollary 3 subsists without metrizability. In fact it characterizes simplexes [2]. One may obtain the second part of Corollary 3 from the first by copying the standard derivation of the Radon-Nikodym Theorem from the Hahn decomposition. This proof, however, invokes the (conditionally $\sigma$-complete) lattice structure of $\mathscr{A}$, and so it depends on the metrizability of $K$.

\section{Faces of class $\mathscr{A}$.}

If $f$ is a positive affine function on a compact convex set $K$, then $f^{-1}(0)$ is seen to be a face. Faces of the form $f^{-1}(0)$ with $f \in \mathscr{A}^{+}$are said to be of class $\mathscr{A}$. 
Theorem 3. The faces of class $\mathscr{A}$ of a metrizable simplex $K$ form a $\sigma$-complete Boolean algebra under the "join" $F, G \frown \operatorname{conv}(F \cup G)$, the "meet" $F, G \frown F \cap G$ and the "complementation" $F \frown F$ ', where $F$ ' denotes the complementary face as defined in [3]. Moreover, the mapping $F \frown F \cap \partial_{e} K$ is a bijection of the class of $\mathscr{A}$-faces onto the class of all Borel subsets of $\partial_{e} K$, and for every Borel subset $A$ of $\partial_{e} K$ with characteristic function $\chi_{A}$ on $\partial_{e} K$, the corresponding $\mathscr{A}$-face is the set $\left(1-\tilde{\chi}_{A}\right)^{-1}(0)$.

Proof. 1) We first prove the second half of the theorem.

If $F$ is an $\mathscr{A}$-face, then $F$ is a fortiori a Borel subset of $K$, and so $\partial_{e} K \cap F$ is a Borel subset of $\partial_{e} K$.

Conversely, let $A$ be a Borel subset of $\partial_{e} K$. By the definition (3.1), $0 \leqq \tilde{\chi}_{A} \leqq 1$, and by Theorem $2,1-\tilde{\chi}_{A} \in \mathscr{A}^{+}$. In particular $F=\left(1-\tilde{\chi}_{A}\right)^{-1}(0)$ is an $\mathscr{A}$-face. Since $\tilde{\chi}_{A}$ is an extension of $\chi_{A}$, we shall have

and

$$
\left(1-\tilde{\chi}_{A}\right)(x)=0 \quad \text { on } \quad \partial_{e} K \cap A
$$

$$
\left(1-\tilde{\chi}_{A}\right)(x)=1 \quad \text { on } \quad \partial_{e} K \backslash A .
$$

It follows that $\partial_{e} K \cap F=A$.

Next we assume that there are two $\mathscr{A}$-faces meeting $\partial_{e} K$ in $A$, or equivalently that there are two functions $f_{1}, f_{2} \in \mathscr{A}^{+}$such that

$$
f_{1}^{-1}(0) \cap \partial_{e} K=f_{2}^{-1}(0) \cap \partial_{e} K=A .
$$

By the barycenter formula (1.1), and by the fact that $\mu_{x}$ vanishes off the $G_{\delta}$-subset $\partial_{e} K$ of $K$, we shall have

$$
f_{i}(x)=0 \Leftrightarrow \int f_{i} d \mu_{x}=0 \Leftrightarrow \mu_{x}\left(\partial_{e} K \backslash A\right)=0,
$$

where $i=1,2$. The right hand term of (4.1) does not depend on $i$, and so $f_{1}^{-1}(0)=f_{2}^{-1}(0)$.

Hence we have proved that $\left(1-\tilde{\chi}_{A}\right)^{-1}(0)$ is the unique face of class $\mathscr{A}$ which meets $\partial_{e} K$ in $A$.

2) Let $f, g \in \mathscr{A}^{+}$. By Theorem $1,(f \wedge g)(x)=0$ if and only if there is a convex combination

$$
x=\lambda y+(1-\lambda) z, \quad 0 \leqq \lambda \leqq 1,
$$

such that $f(y)=g(z)=0$, or equivalently if $x$ belongs to the convex hull of $f^{-1}(0)$ and $g^{-1}(0)$. Hence

$$
\operatorname{conv}\left(f^{-1}(0) \cup g^{-1}(0)\right)=(f \wedge g)^{-1}(0) .
$$

Similarly, $(f \vee g)(x)=0$ if and only if $f(y)=g(z)=0$ for every convex 
combination $(4: 2)$, which in turn is possible if and only if $x$ belongs to the face $f^{-1}(0) \cap g^{-1}(0)$. Hence

$$
f^{-1}(0) \cap g^{-1}(0)=(f \vee g)^{-1}(0) .
$$

Next let $F$ be an $\mathscr{A}$-face of $K$. By the first part of the proof, $F=$ $\tilde{\chi}_{A}^{-1}(1)$, where $\chi_{A}$ is the characteristic function of $A=F \cap \partial_{e} K$ on $\partial_{e} K$. Clearly $\tilde{\chi}_{A}{ }^{-1}(0)$ is an $\mathscr{A}$-face disjoint from $F$. In particular $\tilde{\chi}_{A}{ }^{-1}(0) \subset F^{\prime}$.

By the definition (2.4),

$$
\left(1-\tilde{\chi}_{A}\right) \wedge \tilde{\chi}_{A}=0 .
$$

By Theorem 1, there exists for every $x \in K$ a convex combination (4.2) such that $\tilde{\chi}_{A}(y)=1$ and $\tilde{\chi}_{A}(z)=0$. Thus $K$ is the convex sum of $F$ and $\ddot{\chi}_{A}{ }^{-1}(0)$, which is possible only if $\tilde{\chi}_{A}^{-1}(0) \supset F^{\prime}$. Hence

$$
F^{\prime}=\tilde{\chi}_{A}^{-1}(0) \text {. }
$$

By virtue of (4.3), (4.4), (4.5), the class of $\mathscr{A}$-faces is a Boolean algebra, and it is $\sigma$-complete by the $\sigma$-completeness of $\mathscr{A}$.

By Theorem $3, K$ is (direct) convex sum of $F$ and $F^{\prime \prime}$ for every face $F$ of class $\mathscr{A}$ (cf. [3]). Note also that the mapping $F \frown F \cap \partial_{e} K$ is an isomorphism of the Boolean algebra of $\mathscr{A}$-faces of $K$ onto the Boolean algebra of Borel subsets of $\partial_{e} K$.

TheORem 4. Let $\mu$ be a positive normalized boundary measure on a metrizable simplex $K$. The representing boundary measure $\mu_{x}$ of a point $x \in K$ is absolutely continuous with respect to $\mu$ if and only if $x$ is contained in every $\mathscr{A}$-face containing the barycenter of $\mu$.

Proof. Observe first the following equivalence which is valid for every face $F$ of class $\mathscr{A}$, and which follows by an argument similar to (4.1):

$$
x \in F \Leftrightarrow \mu_{x}(F)=1 \text {. }
$$

Let the barycenter of $\mu$ be denoted by $x_{0}$.

Assume first $\mu_{x} \ll \mu$, and let $F$ be any $\mathscr{A}$-face containing $x_{0}$. By (4.5), $\mu(F)=1$. Hence by absolute continuity $\mu_{x}(F)=1$, and by (4.5) once more, $x \in F$.

Next assume $x \in F$ for every $\mathscr{A}$-face containing $x_{0}$. Let $B$ be any Borel subset of $K$ such that $\mu(B)=0$. By Theorem 3, there is a (unique) face $F$ of class $\mathscr{A}$ such that

$$
F \cap \partial_{e} K=\partial_{e} K \backslash B .
$$

Hence $\mu(F)=1$, and by (4.5), $x_{0} \in F$. By assumption, $x \in F$, and by (4.5) once more $\mu_{x}(F)=1$. It follows that $\mu_{x}(B)=0$. Hence $\mu_{x} \ll \mu$, and the proof is complete. 


\section{REFERENCES}

1. E. M. Alfsen, On the geometry of Choquet simplexes, Math. Scand. 15 (1964), 97-110.

2. E. M. Alfsen, $A$ measure theoretic characterization of Choquet simplexes, Math. Scand. 17 (1965), 106-112.

3. E. M. Alfsen, On the decomposition of a Choquet simplex into a direct convex sum of complementary faces, Math. Scand. 17 (1965), 169-176.

4. E. M. Alfsen, Boundary values for homomorphisms of compact convex sets. (To appear.)

5. H. Bauer, Schilowscher Rand und Dirichletsches Problem, Ann. Inst. Fourier (Grenoble) 11 (1961), 89-136.

6. N. Bourbaki, Intégration, Ch. 1-4 (Act. Sci. et Ind. 1175), Paris 1952.

7. G. Choquet, Existence et unicité des représentations intégrales, Seminaire Bourbaki, Paris, 1956.

8. G. Choquet, Remarques à propos de la démonstration d'unicité de P. A. Meyer, Seminaire du Potentiel, vol. 6, no. 8, Paris, 1962.

9. G. Choquet et P. A. Meyer, Existence et unicité des représentations intégrales dans les convexes compacts quelconques, Ann. Inst. Fourier (Grenoble) 13 (1963), 139-154.

10. D. A. Edwards, Séparation des fonctions réelles définies sur un simplexe de Choquet, C. R. Acad. Sci. Paris 261 (1965), 2798-2800.

11: M. Hervé, Sur les représentations intégrales à l'aide des points extrémeaux dans un ensemble compact convexe métrisable, C. R. Acad. Sci. Paris 253 (1961), 366-368.

12: V. Klee, Convex sets in linear spaces III, Duke Math. J. 20 (1953), 105-112.

13. J. Lindenstrauss, Extension of compact operators, Memoirs Amer. Math. Soc. no. 48 (1964).

14. R. Phelps, Lectures on Choquets theorem (Van Nostrand Mathematical Studies 7), Princeton, N. J., 1966.

15. Z. Semadeni, Free compact convex sets, Bull. Acad. Polon. Sci. 13 (1965), 141-146. 